\author{
Alexandria University \\ Faculty of Arts- English Department \\ Language and Translation Section
}

\title{
A Comparison between Specialized and General Dictionaries With Reference to Legal ones
}

Nada At. Sharaan

Alexandria University 


\begin{abstract}
$\underline{\text { Abstract }}$
Dictionaries play a major role when it comes to different linguistic processes. Indeed, they are quite essential for beginners as they are considered the main sources for obtaining meaning. However, dictionaries do not only provide information related to meanings of words, but they also provide different kinds of linguistic information such as spelling, pronunciation, etymology and syntax. Thus, not only do learners depend on dictionaries, but specialists also consult them for different purposes. In other words, dictionaries have a variety of different functions, that is why Lexicography, which is compiling dictionaries, is quite an essential field.

Dictionary compilers try to make things easier for their users. In fact, the way a dictionary is presented is affected by its purpose or aim. In other words, there are different kinds of dictionaries depending on the type of users. There are general dictionaries and specialized ones. What is the purpose of these types? What are the differences between them? Which is more useful for users? My research is an attempt to provide answers to these questions.
\end{abstract}

Keywords: linguistic, dictionary, general, specialized, user, purpose 


\section{Introduction}

Lexicography is concerned with the way different linguistic information should be presented in a dictionary. Different principles and approaches are created in order to make things easier for learners and specialists. However, things are not that easy as there are different types of users. That is why different types of dictionaries are created. Indeed, it is quite important to compare different types of dictionaries in order to evaluate them. Moreover, it is essential to study the needs of different users. This may help dictionary compilers reach different principles in order to create new and easier dictionaries.

\section{Statement of the Problem}

Dictionaries are consulted by a variety of users for different purposes. Each dictionary is a trial that aims at serving specific purposes. That is why choosing a dictionary to depend on is a challenging task. Moreover, specialized and general dictionaries may contain same lexical items. However, these items are presented in different ways. Thus, it is important for the user to understand the difference between these two types of dictionaries in order to be able to choose the one suitable for his/her needs.

\section{Objectives of the Research}

This research aims to compare between specialized and general dictionaries in order to help the user. Moreover, this research aims to analyze examples from different dictionaries in order to clarify the comparison.

\section{Scope of the Research}

This research is limited to specialized and general dictionaries. Moreover, it is limited to selected examples of monolingual English dictionaries. 


\section{Method of the Research}

This research is an expository one that aims to compare between specialized and general dictionaries. Moreover, an analytical approach is used in order to analyze selected examples.

\section{Review of Literature}

\subsection{General Dictionaries}

The Oxford Handbook of Lexicography is a book, edited by Philip Durken (2016), which discusses different issues related to lexicography. In this book, there is a chapter named "What is a Dictionary for general users.” Indeed, this chapter is written by Henri Bejoint and is dedicated to discussing general dictionaries. Bejoint (2016) says that creating a definition for general dictionaries is a challenging issue. All people understand what a general dictionary is as it is the prototype of the genre (Bejoint, 2016, p. 7). However, when it comes to studying general dictionaries, things are not that easy. He manages to trace the history of dictionaries starting from encyclopedias moving to monolingual dictionaries. He mentions that in the course of the seventeenth century, monolingual dictionaries kept introducing additional words. Indeed, these words were mainly common and functional words. Thus, monolingual dictionaries started to be general ones. In fact, general dictionaries did not appear, early, in Europe because compilers thought that general dictionaries, which contain quite common words, will not be popular among users. Thus they did not want to take the risk as compiling a dictionary is a huge investment (Bejoint, 2016, p. 11). ${ }^{1}$

\footnotetext{
${ }^{1}$ Benjoint, H. (2016). What is a dictionary for general users. In P. Durken (Ed.), The Oxford Handbook of Lexicography. Retrieved from https://books.google.com.eg/books?id=aXXiCgAAQBAJ\&printsec=frontco ver\&dq=The + oxford + handbook + of + lexicography\&hl=en\&sa=X\&ved=0ah UKEwjRysKWvofRAhUQcVAKHa8DDDgQ6AEIGDAA\#v=onepage $\& \mathrm{q}=$ The\%20oxford\%20handbook\%20of\%20lexicograph
} 
He mentions that in the eighteenth and the nineteenth centuries, the number of readers increased in European countries. A lot of books were published and read by the population. Moreover, schools were improved. He says that, at that time, a vast population of readers emerged. Indeed, they belonged to the bourgeoisie which is now known as the middle class. He illustrates that dictionaries were compiled and bought by middle class families as they wanted to improve their level of knowledge and education. Indeed, they wanted to improve their social status through improving their education. Thus, they needed books to help them understand basic grammar and common words. In other words, they needed dictionaries containing basic information and also easy to consult. As a result, different general dictionaries were introduced at that time (Bejoint, 2016, p. 14).

Bejoint (2016) says that general dictionaries have not changed much since the nineteenth century. These dictionaries have played a social role as they represent the language which is quite important for maintain social success. Moreover, these dictionaries are used by all family members as they are considered the source of learning language. However, he mentions that these dictionaries are often dismissed by linguists. This is because they think that these dictionaries are incomplete, simplified, and inaccurate. They also think that these dictionaries are more of commercial objects. Despite of what linguists think, Bejoint mentions that the presence of these dictionaries is a strong evidence of their success. In other words, people, in all countries, depend on these dictionaries as sources of information on words and on things (Bejoint, 2016, p. 24).

\subsection{Specialized Dictionaries}

A Practical Guide to Lexicography is a book, edited by Piet van Sterkenburg (2003), which discusses lexicography in general. In this book, there is a chapter named "Specialized lexicography and specialized dictionaries” written by Lynne Bowker. Bowker (2003) says that specialized lexicography focuses on producing dictionaries that treat specialized fields of knowledge. These dictionaries introduce specific words which are totally different form general words introduced in general dictionaries. Thus, he says that the lexical items introduced in these dictionaries are referred to as terms in order to 
differentiate them from general language words (Bowker, 2003, p. 154). ${ }^{2}$

He illustrates that specialized dictionaries are also called special field dictionaries or special domain dictionaries. This is because they are restricted to a particular subject field. He adds that they can be also called terminological dictionaries or terminological glossaries as they focus on terms (Bowker, 2003, p. 155). He says that specialized lexicography is a branch of lexicography and that is why there is some overlap between the features of specialized and general dictionaries (Bowker, 2003, p. 155). Despite of this fact, he says that specialized and general dictionaries still have different features. For example, they differ in the subject coverage and in the language used. First, he explains that general dictionaries focus on presenting common words, whereas specialized dictionaries focus on terms that are used to describe concepts in specific subject fields. However, he mentions that the difference between common words and specialized terms are not always clear-cut. For example, terms such as HIV or anorexia, once belonged to the medical field, are now used by laypersons. In fact, he mentions that the information general dictionaries present, concerning specialized terms, is less complete than the information presented by specialized dictionaries (Bowker, 2003, p. 156). Second, he talks about the language used in general and specialized dictionaries. He mentions that the microstructure of specialized dictionaries is affected by the language of the dictionary itself. He also says that specialized dictionaries can be monolingual, bilingual, or multilingual (Bowker, 2003, p. 156).

\section{Analysis}

There is a variety of strategies and policies when it comes to compiling a dictionary. These policies depend, mainly, on the type of

\footnotetext{
${ }^{2}$ Bowker, L. (2003). Specialized lexicography and specialized dictionaries. In P. V. Sterkenburg (Ed.), A Practical Guide to Lexicography. Retrieved from https://books.google.com.eg/books?id=xaLuqVY1wfUC\&pg=PA155\&dq= differences+between+general+and+specialized+dictionaries\&hl=en\&sa $=X$ \&ved=0ahUKEwiG 7mUxIXRAhUKIcAKHerGC6wQ6AEIGDAA\#v=one page\&q=differences\%20between\%20general\%20and\%2
} 
users. In other words, dictionary users are the most important factor when it comes to compiling a dictionary. Indeed, the aim of any dictionary is strongly affected by the type of users. Lexicography is concerned with policies and strategies used in compiling different types of dictionaries. In fact, there is no right and wrong when it comes to creating a dictionary. However, dictionaries can be evaluated according to their level of being beneficial for the target users. Dictionaries are consulted by learners, students, university students, specialists, researchers, and so on. Each type has certain needs, that is why there are different types of dictionaries. For example, there are general dictionaries, specialized dictionaries, religious dictionaries and much more. Each type has different aim which affects the way material is presented.

General dictionaries address a wide range of users. Indeed, there is no specific type of users addressed by these dictionaries. This is apparent from the term "general." Compilers of general dictionaries are concerned with presenting the meaning of English lexical items as these dictionaries are mainly consulted for general purposes. Thus, one can claim that there is no specific type of users. That is why general dictionaries tend to present basic definitions of most of the English words. In other words, one can claim that there is no specific criterion a compiler should follow.

On the other hand, specialized dictionaries address a specific type of users; specialists. Each dictionary tackles a certain field. Thus, a specialized dictionary presents all technical terms related to the field tackled. Moreover, the definition tends to be analytical and detailed as it contains technical terminology. In addition, the whole format might be different than that of the general dictionary. This proves that a compiler of a specialized dictionary has, more or less, a specific strategy to follow. Indeed, the most important issue is to be beneficial for specialists of the tackled field. There are medical dictionaries, legal dictionaries, dictionaries for linguistic terms and much more. This paper compares between general and specialized dictionaries with reference to legal ones. 
The first dictionary is Webster's Complete Dictionary of the English Language. ${ }^{3}$ It is a new edition of 1880 with a supplement of new words and an additional appendix of biographical names. Moreover, it is mentioned in the preface that the purpose of this edition is to embrace all the improvements required for the progress of lexicography and language. That is why the dictionary focuses on presenting words that were not presented in previous editions. In addition, this edition aims at collecting new words which are introduced due to the progress of Science and Arts. It is apparent that this dictionary is a general one as it does not focus on a certain field. Instead, it aims at collecting and presenting a variety of English lexical items which belong to different fields and branches.

When one investigates this dictionary, one finds out that this dictionary, like other general dictionaries, is concerned with basic linguistic information which can be useful for all users. For example, it contains a key to the pronunciation in the front matter. This key is explained, in detail, in a later section entitled Principles of Pronunciation. Indeed, pronunciation is an integral issue for users despite of their interests and aims as it shows the user how to pronounce an English word correctly. However, this section is quite detailed, not all general dictionaries have such a detailed section for pronunciation.

When it comes to the entries, the compilers tend to use the alphabetical order which is usually used in most of general dictionaries. Moreover, this dictionary tends to introduce, briefly, some etymological information. This is because general dictionaries aim at presenting all kinds of information which can be beneficial for different users. As a result, they cannot present all information in detail. Thus, it is up to the compilers to choose which information to introduce and which to omit. However, this dictionary tends to introduce different kinds of information. It presents different sections

\footnotetext{
${ }^{3}$ Goodrich, C. A., \& Porter, N. (Eds.). (1886). Webster's Complete Dictionary of the English Language. Retrieved from https://archive.org/stream/websterscomplete00webs\#page/n9/mode/2up
} 
towards the end. There is a section entitled A Pronouncing Biographical Dictionary, which renders the pronunciation of the names of famous figures of different origins, such as, Kerner, Kant and Kaab. There is also a section entitled Pronouncing Vocabulary of Common English Christian Names. Indeed, this section also presents the equivalents of these names in other languages. There are other different sections presented at the end. Thus, this dictionary tends to be comprehensive, to a great extent, as it manages to give the user different accounts of a variety of fields.

When one investigates the entries thoroughly, one finds out that the compilers tend to use analytical definitions. Compilers tend to mention each and every detail related to the lexical item. For example, the lexical item absent is transcribed to show the pronunciation. Then, the French and the Latin versions, of this lexical item, are introduced. Two meanings are explained and followed by quotations of two famous figures; Shakespeare and Chesterfield. The quotations are used instead of using examples. Finally, a synonym is introduced and explained in detail to show the differences. (p. 7) Thus, this shows how the compilers are keen to present a comprehensive interpretation of the lexical item. However, it is important to know that this dictionary is a huge one as it contains 1874 pages. Therefore, the compilers do not face the problem of the size and the space. In fact, this is not the case in all general dictionaries as it is up to the compilers to decide what to mention.

Things are different when it comes to specialized dictionaries. It is mentioned before that these dictionaries aim at addressing a certain field. Thus, this affects choice of words, way of presenting and way of defining. Black's Law Dictionary is a legal dictionary which contains definitions of terms and phrases of American and English Jurisprudence. The compiler is Henry Campbell Black who is a specialist in the field of law. The edition used, in this paper, is a fourth revised edition (1968). It is obvious, from the title, that this dictionary aims at explaining legal terms. Thus, all lexical items, included in the

\footnotetext{
${ }^{4}$ Black, H. C. (1968). Black's Law Dictionary. Retrieved from

http://heimatundrecht.de/sites/default/files/dokumente/Black'sLaw4th.pdf
} 
dictionary, are mainly legal. Moreover, it is obvious that this dictionary should be consulted for legal purposes and by specialists, otherwise it will be unbeneficial. It is mentioned in the preface that this edition is made because the law has undergone substantial changes and developments. That is why some revisions and additions are included in this edition. Moreover, it is mentioned in the preface that a Guide of Pronunciation is introduced in the front matter and a table of Abbreviations is presented at the end of the volume. In addition, the front matter contains Code of Professional Responsibility and Canon of Judicial Ethics. These sections are quite important for students of law not for all students and users.

The Compiler uses the alphabetical order but in a totally different way. In this dictionary, the term is written on the top of the page and it is defined according to different aspects. For example, the term abandonment is defined in almost 6 pages. The term is written at the top of each page, and then it is defined according to different aspects, such as, Employment, Homesteads, Children and much more. The term is associated with these fields and explained from a legal point of view. In other words, it is as if the term is explained according to the context concerning legal issues. Indeed, it is totally different from what is used in general dictionaries. Moreover, each definition is followed by the source used by the compiler because, in legal issues, sources are essential. For example, the term abandonment is defined, under Perspective Rights as follows:

\section{Perspective Rights}

Non-use alone is sufficient. Burkman v. City of New Lisbon, 246 Wis.547, 19 N. W.2d 311, 313; Smelcer v. Rippetoe, 24 Tenn. App. 516, 147 S.W.2d 109, 113, 114 , (p.13).

The lexical item acceptance is mentioned in both dictionaries differently. First, in Webster's Complete Dictionary of the English Language, some phonetic signs are used in order to show the accurate pronunciation. The dictionary renders a general meaning of the lexeme with an example. Indeed, in this general definition, the word class is mentioned. Then, the general definition is followed by five different senses. These senses differ according to the field in which the word is 
used. For example, this lexical item in legal context has a different meaning. This attitude is not followed in all general dictionaries; some of them only mention the most used senses without referring to different fields. Indeed, this dictionary manages to collect different meanings of the same lexeme to a great extent. This proves that there is no fixed criterion to be followed when it comes to the type of information introduced in general dictionaries.

Second, in Black's Law Dictionary, the term acceptance is explained in 2 pages. The compiler gives a general definition of the term at the beginning. Then, the term is explained under different legal aspects. Indeed, these aspects are alphabetically organized. It is explained under Bills of Exchange, Insurance, Marine Insurance, Sales, Civil Law, Pleading, and Canon Law. In other words, the term is tackled, from a legal point of view, in different aspects and situations. One may think that it is quite an easy and an organized way of presenting the material. However, it does not introduce any linguistic information. Thus, this dictionary is to be consulted, only, by specialists and law students for legal purposes.

It is obvious, from what is mentioned, that there are a lot of differences between general and specialized dictionaries. Indeed, one feels that compilers have more freedom when it comes to compiling general dictionaries. This is because they are the ones who decide on the type of information to be mentioned. In our case, the compilers tend to make the dictionary, more or less, a comprehensive one. In fact, this is not a must for all general dictionaries. On the other hand, one feels that compilers of specialized dictionaries do not have much freedom. This is because these dictionaries should present all types of information needed by the users. However, when it comes to the way of presenting the material in the two types of dictionaries, one feels that it is up to the compilers as long as they make it clear to the user how to use it. Thus, there is no specific criterion to be followed when it comes to presenting the material in both dictionaries. 


\section{Findings of the Research}

There are a lot of differences between general and specialized dictionaries. First, the type of information, in general dictionaries, is, more or less, linguistic as these dictionaries endeavor to present the meaning of most of English words. Moreover, these dictionaries focus, mainly, on pronunciation, syntax, etymology, semantics and much more. On the other hand, specialized dictionaries are concerned with all terms that are related to the field. They also focus on explaining the terms with reference to the field and that is why they do not introduce any linguistic information except if they are tackling the linguistic field.

Second, the way of presenting, in the two types, is different. General dictionaries tend to use the alphabetical order and all entries are introduced under each other. However, in specialized dictionaries, the way of presenting may depend on the aspects used to define as it is mentioned in the dictionary discussed in the paper.

Finally, one may claim that compilers of general dictionaries have more freedom as they are the ones who choose the information to be mentioned. This is because they do not address specific users. However, one do not feel that compilers of specialized dictionaries have this kind of freedom as they should, in a way or another, mention all terms that belong to the field. 
